\title{
Artigo de Reisão
}

\section{ADESÃO AO TRATAMENTO, ACESSO E QUALIDADE DA ASSISTÊNCIA EM AIDS NO BRASIL}

\author{
Maria Inês Batistella Nemes*1, Elen Rose lodeiro Castanheira², Ernani Tiaraju de Santa Helena ${ }^{3}$,Regina Melchior ${ }^{4}$, Joselita Magalhães Caraciolo ${ }^{5}$, Cáritas \\ Relva Basso ${ }^{6}$, Maria Teresa Seabra Soares de Britto e Alves ${ }^{7}$, Tatianna Meireles Dantas de Alencar ${ }^{8}$, Dulce Aurélia de Souza Ferraz ${ }^{9}$ \\ Trabalho desenvolvido no Departamento de Medicina Preventiva, Faculdade de Medicina da Universidade de São Paulo e outras instituições as quais \\ pertencem os integrantes da Equipe QualiAids, formada por pesquisadores de universidades brasileiras e profissionais do Programa Nacional de DST e AIDS
}

* Correspondência:

Maria Ines Batistella Nemes

Av Dr. Arnaldo, 450

Cerqueira César

São Paulo - SP

CEP: 01246-903

Tel. 551130617078

e-mail: mibnemes@usp.br

\begin{abstract}
RESUMO
A adesão à terapia antirretroviral (TARV) é crucial para a efetividade e o impacto do tratamento da Aids. Este artigo discute as relações entre adesão e qualidade dos serviços de assistência a pessoas vivendo com Aids (PVA), evidenciando a qualidade como elo central entre adesão e acesso. Está baseado nos resultados de pesquisas que conduzimos sobre a atenção a PVA no Brasil. Nossos estudos apontam que os grupos de pacientes acompanhados em serviços com número inferior a 100 pacientes apresentam risco estimado de não adesão maior do que os grupos acompanhados em serviços com mais de 500 pacientes. Apontam também que serviços com menos de 100 pacientes têm risco estimado maior de pertencer a grupos de má qualidade. Isto está relacionado à baixa complexidade observada nos serviços de menor porte caracterizada por: dificuldades em manter uma estrutura mínima de recursos humanos e materiais, simplificação da organização dos processos de trabalho, centramento no trabalho autônomo do profissional médico e gerenciamento sem projeto técnico. Há necessidade de pautar novos estudos sobre adesão e qualidade. As evidências existentes já apontam, porém, a necessidade de revisão na alocação dos serviços de assistência a PVA, bem como a de homogeneizar a qualificação destes serviços, condições necessárias para a manutenção de taxas aceitáveis de adesão à TARV no país.
\end{abstract}

Unitermos: Aids. Terapia antirretroviral de alta potência. Adesão ao tratamento. Qualidade da assistência à saúde.

\section{INTRODUÇão}

O sucesso da terapia antirretroviral de alta potência para a Aids (TARV) depende da manutenção de altas taxas de adesão do paciente ao tratamento medicamentoso ${ }^{1,2}$. Analisar os fatores associados à adesão é fundamental para a melhoria das políticas e práticas de saúde voltadas ao aprimoramento da efetividade do tratamento. Com o objetivo de contribuir neste sentido, procuramos neste artigo discutir relações entre adesão, qualidade e acesso aos serviços de saúde tendo por base os resultados de pesquisas avaliativas que nosso grupo de pesquisa, a Equipe QualiAids, desenvolveu nos últimos anos sobre adesão à TARV e qualidade dos serviços ambulatoriais para PVA no Sistema Ú nico de Saúde do Brasil (SUS). A Equipe QualiAids é formada por docentes e pesquisadores de várias universidades brasileiras. Dedica-se a estudos avaliativos sobre qualidade e adesão ao tratamento da Aids e de outras doenças crônicas no SUS.

0 artigo discute implicações dos resultados de duas pesquisas. A primeira foi conduzida entre 1998/99 em 27 serviços do Estado de São Paulo que assistiam, em nível ambulatorial, 8.550 pessoas vivendo com Aids (PVA) sob tratamento

1. Professor do departamento de Medicina Preventiva, Faculdade de Medicina da UNESP; Coordenadora da Equipe QualiAids, São Paulo,SP

2. Professor do Departamento de Saúde Pública, Faculdade de M edicina da UNESP; Campus de Botucatu, Integrante da Equipe QualiAidS, São Paulo,SP

3. Professor do departamento de Medicina, Universidade Regional de Blumenau; Integrante da Equipe QualiAids. Blumenau, SC

4. Professor do departamento de Saúde Coletiva da UEL;Integrante da Equipe QualiAids. Londrina, PR

5. Medica infectologista do Programa de DST e Aids da Secretaria de Estado da Saúde de São Paulo; Pós- graduanda do departamento de Medicina Preventiva da Faculdade de Medicina da USP; Integrante da Equipe QualiAids, São Paulo,SP

6. Médica infectologista do Programa de DST e Aids da Secretaria de Estado da Saúde do Estado de São Paulo; Pós-graduanda do departamento de Medicina Preventiva da Faculdade de Medicina da USP; Integrante da Equipe QualiAids, São Paulo,SP

7. Professor do departamento de Saúde Pública da UFMA; Integrante da Equipe QualiAids, São Luis-MA

8. Técnica do Programa Nacional de DST e Aids do Ministério da Saúde; Integrante da Equipe QualiAids, Brasília, DF

9. Técnica do Programa Nacional de DST e Aids do Ministério da Saúde; Pós-graduanda do departamento de Medicina Preventiva da Faculdade de Medicina da USP; Integrante da Equipe QualiAids, São Paulo,SP 
Nemes MiB et al.

antirretroviral. Envolveu abordagens quantitativas e qualitativas sobre adesão à TARV e sobre características dos serviçOS $^{3,4,5}$. A segunda, conduzida entre 2000/03, envolveu 0 universo dos serviços de sete Estados que assistiam 92.399 pacientes em TARV. A primeira fase $\mathrm{e}^{6,7,8}$ classificou os serviços em quatro níveis de qualidade e a segunda fase ${ }^{9}$ analisou a adesão dos pacientes destes serviços ao tratamento ${ }^{4}$.

\section{A adesão é (também) resultado da qualidade do serviço}

Ao desenhar o primeiro estudo que realizamos sobre adesão à TARV em $1998 / 9^{3}$, além de características dos pacientes, da doença e do tratamento, questões tradicionalmente investigadas em estudos sobre o tema, incluímos características dos serviços de saúde como possíveis fatores explicativos da adesão. Esta assunção teve como base trabalhos sobre adesão ao tratamento de várias doenças que a associavam a características da assistência prestada, particularmente aos aspectos de acessibilidade e da relação médico-paciente ${ }^{10-13}$.

A partir dos dados obtidos, os 27 serviços do Estado de São Paulo participantes do estudo foram agregados segundo grau de complexidade (definido pelo número de pacientes sob TARV e número de modalidades assistenciais existentes além da ambulatorial: assistência domiciliar, hospital-dia e/ou internação) e segundo indicadores selecionados de qualidade: disponibilidade de recursos humanos, medicamentos e exames; controle de abandono e de faltas; e opinião do gerente sobre 0 desempenho da equipe ${ }^{3}$. A adesão à TARV foi avaliada mediante questionário aplicado a uma amostra aleatória de 1141 pacientes dos serviços participantes.

Observamos que o agregado de unidades com baixa complexidade assistencial e de piores indicadores de qualidade apresentou um risco estimado para não-adesão quatro vezes maior (com intervalo de confiança de $95 \%$ entre 2,0 e 8,4 ) do que 0 agregado de alta complexidade assistencial e melhores indicadores de qualidade. Este resultado foi obtido na análise multivariada ajustada por escolaridade, salário, tempo do diagnóstico de infecção pelo HIV, consumo abusivo de bebida alcoólica, falta na consulta médica (variáveis estas que se mostraram associadas na análise univariada e permaneceram no modelo final) ${ }^{3}$.

Analisando esta associação, vimos que a qualidade foi mais importante que a complexidade para determinar a associação entre 0 agregado de serviços e a adesão. Mas vimos também que a complexidade do agregado, determinada, sobretudo, pelo número de pacientes sob TARV, estava fortemente correlacionada com a qualidade, isto é, serviços com maior número de pacientes tenderam a apresentar também melhores indicadores de qualidade.

Interessante para confirmar a relação entre qualidade do serviço e adesão, este resultado, entretanto, colocou a seguinte questão: haverá qualidade independentemente da complexidade? Ou, em outros termos, é possível ter serviços com número pequeno de pacientes, menos complexos, de boa qualidade e com boas taxas de adesão? 0 desenho utilizado, com poucos indicadores de qualidade, não pareceu suficiente para responder com maior acurácia a estas questões. Com este objetivo, desenhamos a nova pesquisa sobre adesão e qualidade dos serviços iniciada em 2000.
Figura 1 - Serviços agrupados segundo k-médias de indicadores de qualidade

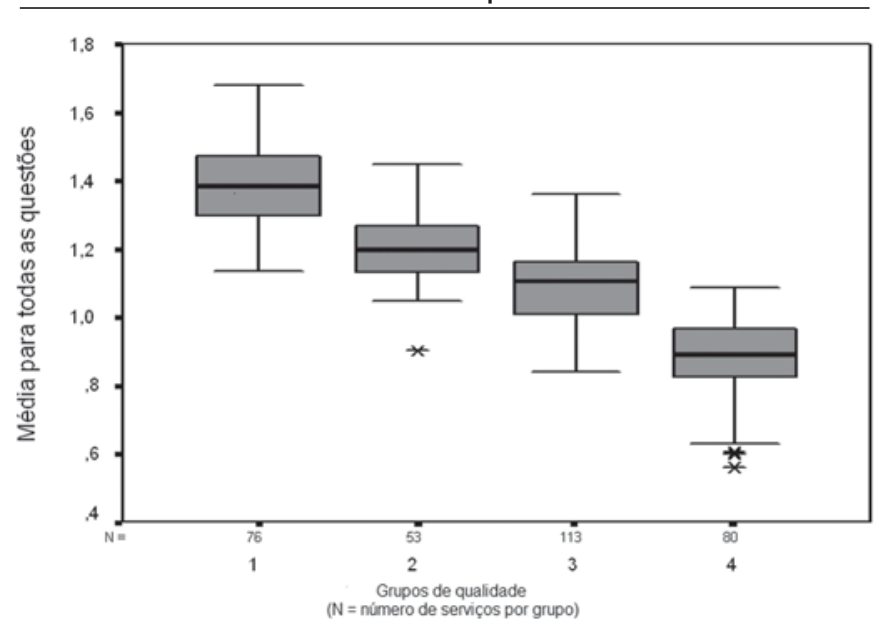

* Extraída de Melchior R. Avaliação da Organização da Assistência Ambulatorial A Pessoas Vivendo Com Hiv/Aids no Brasil. Análise de 322 serviços em 7 estados brasileiros (CE, MA, MS, PA, RJ , RS, SP. [Tese de doutorado]. São Paulo: Faculdade de Saúde Pública, Universidade de São Paulo, 2003

\section{A qualidade dos serviços está associada ao seu grau de complexidade. 0 grau de complexidade está associado à adesão.}

Esta nova pesquisa foi desenvolvida em sete Estados brasileiros (CE, MA, MS, PA, RJ, RS, SP). Estes estados, na ocasião, concentravam $63 \%$ dos serviços de Aids do país e acompanhavam $72 \%$ do total de PVA sob TARV no Brasil. A avaliação da qualidade dos serviços ${ }^{6,7,8,14}$ precedeu a análise da adesão.

Os serviços foram avaliados mediante um questionário pré-testado e validado, respondido pelos gerentes, que continha 106 indicadores de qualidade referentes à estrutura e ao processo da assistência. Cada um dos indicadores foi classificado segundo uma escala de 0,1 e 2, sendo o valor 2 correspondente à melhor classificação Para que a complexidade do serviço não influenciasse a medida de qualidade, admitiu-se, em primeiro lugar, uma disponibilidade mínima de recursos que todos os serviços deveriam ter (por exemplo, suprimento sem interrupção dos ARV utilizados na primeira linha de tratamento). A partir deste mínimo, todos os incrementos na disponibilidade de recursos humanos e materiais foram avaliados de modo proporcional ao número de pacientes (por exemplo, o serviço que conta com um médico para 150 ou menos pacientes recebeu a avaliação máxima neste item). A média entre 0 e 2 obtida pelo conjunto de indicadores de qualidade classificou os serviços em um ranking de qualidade ${ }^{7,8}$.

Para avaliar o perfil de qualidade do conjunto dos serviços elegemos uma técnica estatística de agrupamento, de modo a permitir a formação do menor número possível de grupos de qualidade heterogêneos. A técnica possibilitou agregar os serviços em quatro agrupamentos de nível de qualidade estatisticamente diferentes. Agruparam-se nos melhores níveis $40 \%$ dos serviços (76 serviços no nível 1 e 53 no nível 2) 
Adesão ao tratamento, acesso e qualidade da assistência em Aids no Brasil

e $60 \%$ nos piores níveis ( 113 serviços no nível 3 e 80 no nível $4)^{8}$. (Figura 1)

Analisamos, então, a associação entre pertencimento aos melhores grupos de qualidade e as seguintes características institucionais dos serviços: tempo de funcionamento, porte do município, número de pacientes e exclusividade do serviço (totalmente especializado em DST/Aids ou inserido em outra estrutura do SUS). Mantiveram-se no modelo final as variáveis: porte do município, número de pacientes e exclusividade do serviço.

Para os grupos de serviços com mais de 500 pacientes 0 risco ajustado estimado pela razão de chances de pertencer aos grupos de melhor qualidade foi 3,4 vezes maior (com intervalo de confiança de $95 \%$ entre 1,5 e 7,7 ) do que nos de unidades com menos de 100 pacientes. E foi 7,5 vezes maior (com intervalo de confiança de $95 \%$ entre 3,0 e 19,0 ) nos serviços exclusivos em relação àqueles inseridos em outras estruturas assistenciais do SUS ${ }^{8}$. (Tabela 1 )

Note-se que a proporção de serviços exclusivos foi pequena - 13\%, dos quais a maioria são centros de referência localizados em grandes municípios - enquanto a proporção de serviços com menos de 100 pacientes foi alta: 48,8\%. Mesmo Estados do Sul e do Sudeste contavam com número considerável de serviços com menos de 50 pacientes: no Rio Grande Sul, 13 (41\%), no Rio de Janeiro, 34 (31\%) e, em São Paulo, $26(27 \%)^{8}$.

Após a avaliação da qualidade, medimos a adesão mediante questionário aplicado a uma amostra aleatória de 1972 pacientes dos serviços avaliados quanto à qualidade. Embora a adesão tenha sido menor entre os grupos acompanhados em serviços agrupados nos piores níveis de qualidade, esta diferença não foi significativa. ${ }^{9}$.Provavelmente, nossa opção em agregar os serviços no menor número possível de grupos de qualidade, que resultou em apenas quatro grupos para 322 serviços, tornou insuficiente o poder do estudo para detectar diferenças entre as prevalências de adesão dos quatro grupos.

Entretanto, quando analisamos a associação entre adesão e características de complexidade dos serviços, vimos que os grupos de pacientes tratados em serviços com número menor de 100 pacientes mostraram risco estimado bruto de não-adesão significativamente maior que os serviços com mais de 500 pacientes (serviços com menos de 100 pacientes: OR bruto $=$ 1,53). Quando ajustado segundo o nível de qualidade, o risco dos serviços com menos de 100 pacientes aumentou. Com 0 nível de qualidade controlado na análise, serviços com número menor de pacientes mostraram risco estimado de não-adesão 1,73 vezes maior do que unidades com mais de 500 pacientes (IC95\% ajustado: 1,5-7,7) ${ }^{9}$. (Tabela 2)

Assim, grupos de pacientes acompanhados em serviços com menos de 100 pacientes têm maior risco estimado de nãoadesão. Se, entretanto, o serviço pertencer a um grupo de boa qualidade, este risco é menor. Entretanto, como acima mostrado, a probabilidade de um serviço com número pequeno de pacientes pertencer a um grupo de boa qualidade é muito menor do que a de um serviço mais complexo.

Tentemos entender melhor isto. 0 número pequeno de pacientes não é uma dimensão de qualidade em si. Ele se mostrou um ótimo indicador de dimensões da qualidade. E de quais dimensões? Das consideradas na análise, ou seja, possuir ao menos a disponibilidade de recursos considerada mínima e
Tabela 1 - Odds Ratio ajustado de pertencer aos grupos de serviç de melhor qualidade (Grupos 1 e 2 ) segundo número de pacientes e exclusividade do serviço

\begin{tabular}{lc}
\hline Variáveis & OR $\left(\right.$ IC $\left._{95 \%}\right)$ \# \\
\hline No pacientes & \\
Até 100 & 1,0 \\
101 a 500 & $3,0(1,6-5,4)$ \\
Mais de 500 & $3,4(1,5-7,7)$ \\
Exclusividade & 1,0 \\
Não exclusivo & $7,5(3,0-19,0)$ \\
Exclusivo DST/AIDS & \\
\hline
\end{tabular}

Ajustado segundo grupo de município

* Extraída de Melchior R. Avaliação da Organização da Assistência Ambulatorial A Pessoas Vivendo Com Hiv/Aids no Brasil. Análise de 322 serviços em 7 estados brasileiros (CE, MA, MS, PA, RJ , RS, SP. [Tese de doutorado]. São Paulo: Faculdade de Saúde Pública, Universidade de São Paulo, 2003

Tabela 2 - Odds Ratio bruto e ajustado para não-adesão a TARV segundo número de pacientes do serviço

\begin{tabular}{lcc}
\hline Variáveis & OR & OR (IC $\left.{ }_{95 \%}\right) \#$ \\
\hline $\begin{array}{l}\text { No pacientes } \\
>500\end{array}$ & 1,0 & \\
301 a 500 & 0,92 & $0,90(0,65-1,25)$ \\
101 a 300 & 1,14 & $1,18(0,89-1,53)$ \\
$<100$ & 1,53 & $1,73(1,13-2,64)$ \\
\hline
\end{tabular}

Ajustado segundo grupo de qualidade

* Extraída de Nemes MIB, Carvalho HB, Souza MFMS. Antiretroviral therapy adherence in Brazil. AIDS. 2004; 18 (Suppl 3): 5-20

ter uma adequada organização do processo de trabalho assistencial.

Os serviços de menor porte têm mais dificuldade para reunir e manter equipes mínimas. Frequentemente têm processos de trabalho simplificados, centrados quase totalmente no trabalho autônomo do profissional médico, que, em geral, acompanha pacientes com muitas outras patologias. 0 gerenciamento é, em geral, centrado no cumprimento de formalidades - e a atuação dos profissionais não é supervisionada nem discutida. Assim, por exemplo, o relato de existência de reuniões técnicas, discussão e padronização de condutas foi praticamente nulo entre os serviços de menor porte ${ }^{6}$.

Evidentemente, existem serviços de menor porte que, por força da adequada provisão de recursos materiais e tecnológicos e da qualidade técnica e compromisso de seus profissionais, alcançam bons níveis de qualidade. E que, por outro lado, vários serviços complexos não os alcançam ${ }^{15}$. Entretanto, as possibilidades de um serviço complexo de superar condições adversas de aporte de recursos e de tecnologias são muito maiores, como bem mostra uma mínima observação da estrutura e história da rede de serviços do sistema de saúde no Brasil.

\section{0 que (ainda) não sabemos sobre adesão e qualidade: perspectivas para novos estudos}

Sabemos ainda pouco sobre adesão e urge saber mais ainda sobre a qualidade da assistência. 
Não sabemos a prevalência atual da adesão. 0 último estudo de abrangência nacional foi o que realizamos em $2002^{9}$. Também a influência na adesão de fatores emergentes, como a lipodistrofia, ainda pouco abordada no Brasil ${ }^{16,17}$, necessita de mais investigação.

Adicionalmente, a maioria dos estudos sobre adesão desenvolvidos até hoje no Brasil são, tais como as nossas pesquisas, estudos epidemiológicos de corte transversal ${ }^{18-22}$. Estudos de corte longitudinal são raros e restritos a poucos sítios ${ }^{23,24}$. A adesão varia no tempo e o estudo de fatores de risco ganha poder explicativo nos estudos longitudinais, que permitem monitorar as variações da adesão ao tratamento em indivíduos e, destes, agregados por serviços ${ }^{25}$.

O Programa Nacional de DST e Aids do Brasil (PN DST/Aids) vem, desde o final dos anos 90 , sistematicamente recomendando que a adesão seja considerada item prioritário nas atividades de assistência dos serviços do SUS que atendem PVA. A adesão tem sido ainda tema recorrente em eventos científicos e encontros profissionais do campo da Aids no Brasil e nas publicações e atividades da sociedade civil organizada. Em todos estes campos, a implementação de tecnologias de processo focadas na adesão é enfaticamente recomendada, merecendo especial destaque a constituição de "grupos de adesão" 26.

Embora gerentes e dirigentes dos vários níveis de gestão confirmem a implementação de tecnologias de adesão em muitos serviços, não há avaliação disponível sobre a efetividade, o custo ou sequer a cobertura dessa e de outras tecnologias voltadas para a adesão. $\mathrm{Na}$ pesquisa sobre qualidade conduzida entre $2000 / 2001,18,3 \%$ (59) dos serviços informaram conduzir rotineiramente "grupos de adesão" . Não há porém caracterização disponível deste grupos que podem ter várias orientações: educativos, operativos, de reforço comportamental, etc. Em revisão da literatura, encontramos apenas dois estudos publicados recentemente que avaliam intervenções de adesão em grupos reduzidos de pacientes 27,28 .

Em relação à qualidade, embora novos e interessantes aportes tenham sido abordados em estudos recentes ${ }^{29}$ falta atualizar a avaliação da qualidade dos serviços: a avaliação mais abrangente foi a que realizamos em 2000/2001.6 Nesta direção, o Questionário QualiAids ${ }^{30}$, ferramenta eletrônica de avaliação que construímos em 2005 com base na nossa pesquisa sobre qualidade, poderá contribuir. A ferramenta permite a auto-avaliação dos serviços, acompanhada de recomendações de boas práticas ${ }^{31}$. Foi recentemente aplicada ao conjunto dos serviços do Programa Nacional de DST e Aids ${ }^{32}$. Entretanto, novos e diversos aspectos da qualidade precisam ainda ser estudados, também no sentido de aprofundar 0 entendimento sobre suas relações com a adesão.

Nesses últimos anos, nossa equipe vem trabalhando em projetos identificados com essas necessidades de investigação. Cremos que estas e várias outras devem merecer destaque na agenda de pesquisa das instituições que compõem a resposta brasileira à Aids. Certamente é preciso saber mais sobre adesão e qualidade. Contudo, o que já se sabe parece suficiente para instruir algumas recomendações potencialmente positivas para a melhoria imediata da efetividade da assistência às PVA no Brasil.

\section{Ampliar a efetividade do tratamento: qualificar o acesso}

Dada a amplitude e as dificuldades de acesso geográfico em certas regiões com baixa prevalência de Aids, é mandatória a instalação de serviços nesses locais, mesmo que seja para assistir a pequeno número de pacientes. Para reduzir as chances de não-adesão e de má qualidade, é necessário que esses serviços estejam de fato integrados a outros maiores e mais especializados. Isto significa que entre eles deve existir estreita relação, que possibilite a superação do funcionamento tradicional do sistema de referência e contrarreferência e a estruturação de um sistema de "pupilo-mentor", no qual, além da mútua referência de pacientes, haveria também a permuta de conhecimento. Deste modo, os serviços de maior complexidade poderiam atuar como "tutores" e auxiliar o desenvolvimento e capacitação dos profissionais e gerentes dos serviços de menor complexidade ${ }^{31}$.

A distribuição dos serviços que encontramos no cadastramento realizado para a pesquisa em 2000 mostrou que, entre os Estados com alta prevalência de Aids, a razão geral entre pacientes e serviços guardava certa proporção. 0 Estado de São Paulo, por exemplo, com quase $40 \%$ dos doentes contava com $30 \%$ do total de serviços. Nos Estados de baixa prevalência, essa razão foi muito mais desproporcional. Estados da região amazônica, com aproximadamente $10 \%$ dos pacientes, contavam com apenas $2 \%$ do total de serviços. Por outro lado, estados do Sudeste e Sul, onde não é possível dizer que dificuldades de acesso geográfico justificariam a instalação de microsserviços, os têm mantido em proporções importantes como vimos acima ${ }^{6}$.

Não encontramos nas bases de dados disponíveis levantamentos atualizados da distribuição entre serviços e pacientes. As evidências indicam que a análise desta distribuição é crucial. Se há acordo sobre a necessidade de ampliar o acesso à assistência às PVA em todas as regiões do Brasil, é vital que se consolide mais 0 acordo sobre a necessidade de ampliar 0 acesso com qualidade.

\section{Ampliar a efetividade do tratamento: qualificar o gerenciamento dos serviços}

Nos estudos que realizamos, as seguintes variáveis mostraram-se associadas a maiores chances de não-adesão: baixa escolaridade, baixa renda, uso atual e abusivo de álcool, número grande de comprimidos no esquema ARV e faltas no seguimento $3,4,9$. Estas associações são consistentes com estudos nacionais realizados em contextos 10cais $^{21,22,32,33}$ com vários estudos internacionais ${ }^{34,35,36}$ e estão bem estabelecidas.

A avaliação dos serviços mostrou, entretanto, que os fluxogramas de assistência em grande parte das unidades são rotineiros, iguais para todos os pacientes, não priorizando os grupos de maior risco de não-adesão ${ }^{8}$. É preciso lembrar, contudo, que as associações encontradas referem-se a grupos com maiores chances de não-adesão, o que não significa que as pessoas pertencentes a estes grupos terão necessariamente menos adesão. Criar fluxogramas especiais para grupos não substitui o cuidado individual e, sobretudo, não deve implicar atitudes discriminatórias com aqueles erroneamente chamados "pacientes de risco" para não-adesão como, lamentavelmente, 
tem sido constatado em alguns contextos ${ }^{4,37}$. Trata-se, ao contrário, de oferecer cuidado especial àqueles com maior vulnerabilidade, justamente na direção de promover ativamente a equidade.

\section{Conclusão}

A complexidade da TARV foi, por muito tempo, utilizada como argumento contra a expansão do tratamento da Aids em países pobres, presumivelmente "sob maior risco" de não-adesão $0^{38,39}$. O Brasil foi o primeiro país não rico a adotar a política de acesso universal e gratuito à TARV. Por essa razão, nosso estudo de $2002^{9}$ teve importante efeito-demonstração $0^{40,41}$ ao evidenciar que a despeito do perfil socioeconômico das PVA no Brasil, caracterizado por baixa renda e baixa escolaridade, a prevalência de adesão obtida $(75 \%)$ foi semelhante às obtidas em estudos provenientes de países ricos à época. Este fato se somou aos inegáveis sucessos da resposta brasileira à epidemia de Aids ${ }^{42}$. Estes sucessos, porém, precisam ser mantidos e ampliados. Lidamos agora com uma epidemia heterogênea e em mutaçã $0^{43}$ e com um número grande de pessoas ainda não diagnosticadas. O número de pessoas que precisará de tratamento constante tende a aumentar com o desejável aumento de cobertura da testagem do HIV. Garantir tratamento acessível e de boa qualidade para este grupo exige arranjos institucionais e tecnologias específicas e especializadas, dada a heterogeneidade epidemiológica e de aporte de recursos no Brasil.

Os princípios do SUS de descentralização e regionalização devem constituir a diretriz geral destas iniciativas. Ressalte-se, porém, que, estes princípios destinam-se a orientar ações de saúde adequadas às realidades locais. 0 que quer dizer que, evidentemente, a estruturação da atenção deve, antes e mais do que atender necessidades institucionais, atender necessidades de saúde.

Neste raciocínio, doenças de alta prevalência e distribuídas homogeneamente devem ser abordadas por estruturas de atenção extensivas, como a da atenção básica. Este é tipicamente o caso de doenças como a hipertensão arterial não complicada. Doenças de baixa prevalência e de distribuição heterogênea, como a Aids, exigem abordagem diferenciada. A estas características epidemiológicas, se acresce a ainda existente dificuldade no manejo clínico da patologia ao lado de suas peculiaridades sociais e psicológicas. Neste caso, sempre que possível, a necessidade de qualidade - o que, aqui, quer dizer complexidade - deve prevalecer sobre a necessidade de acesso extensivo. Coerentemente com aqueles mesmos princípios, também esta não pode ser tomada como diretriz absoluta. 0 acesso universal e livre ao tratamento da Aids constitui a principal base técnica e ética da assistência aos que vivem com Aids no Brasil. Mantê-lo, mesmo que "simplificado", é uma solução temporária aceitável para certas regiões. Será sempre preciso lembrar, entretanto, que soluções mais perenes passam pelo incremento de recursos que possibilitem 0 aumento da capacidade instalada e da qualificação de todos os serviços. Não manteremos em longo prazo taxas aceitáveis de adesão ao tratamento da Aids sem que estas condições sejam asseguradas.

\section{Agradecimentos}

Mary Franklin Gonçalves que nos auxiliou nas pesquisas da Equipe QualiAids; aos profissionais e usuários dos serviços de saúde participantes das nossas pesquisas.

\section{Conflito de interesse: não há}

\section{SUMmary}

The patient adherence to highly active antiretroviral therapy (HAART) is a crucial matter to AIDS treatment effectiveness and its' impact. This article aims to discuss the association between adherence and quality of health service providing care to people living with AIDS (PLWA), highlighting quality of the services as a central point to adherence and access. It is based on results of our previous studies about the health care to PLWA in Brazil. Our studies point out that the groups of patients who are followed-up in health services providing care for less than 100 patients presented greater estimated risk of non-adherence than services following more than 500 patients. Also, smaller health services showed greater estimated risk to be ranged in the worst quality of services groups. This is related to the low complexity of smaller health care services, such as: lack of minimum human resources and material structures, poor organization on work process, medical-centered care and poor technical management. New studies in adherence and quality of services are needed. Nevertheless, the existent findings have already pointed out the need to review the current distribution of AIDS care services as well as to make the quality of services more homogenous thorough the country. These are high priorities in order to keep acceptable levels of adherence to HAART in Brazil. [Rev Assoc Med Bras 2009; 55(2): 207-12]

KEY WORDS: AIDS. Antiretroviral therapy. Highly Active. Adherence. Quality of health care

\section{ReferênCIAS}

1. Bangsberg DR, HechtFM, Charlebois ED, Zolopa AR, Holodniy M, Sheiner L, et al. Adherence to protease inhibitors, HIV-1 viral load, and development of drug resistance in an indigent population. AIDS. 2000;14(4):357-66.

2. Paterson DL, Swindells S, Mohr J, Brester M, Vergis EN, Squier C, et al. Adherence to protease inhibitor therapy and outcomes in patients with HIV infection. Ann Intern Med. 2000;133(1):21-30.

3. Nemes MIB, organizador. Avaliação da aderência ao tratamento por antiretrovirais em usuários de ambulatórios do sistema público de assistência à AIDS no Estado de São Paulo. Brasília (DF): Coordenação Nacional DST/AIDS, Ministério da Saúde; 2000 . (Série Avaliação No 1). Disponível em http://www.Aids.gov.br/ avalial/home.htm.

4. Mesquita F, Doneda D, Gandolfi D, Nemes MIB, Andrade T, Bueno R, Trigueiros DPE. Brazilian Response to the Human Immunodeficiency Virus/Acquired Immunodeficiency Syndrome Epidemic among Injection Drug Users. Clin Infect Dis. 2003;37(Suppl.5):S382-5.

5. Melchior R, Nemes MIB, Alencar TMD, Buchalla CM .Challenges of treatment adherence by people living with HIV/AIDS in Brazil. Rev Saúde Pública. 2007;41(Supl 2).

6. Nemes MIB, Castanheira ERL, MelchiorR, Basso, CR, Alves, MTSSB. Avaliação da Qualidade da Assistência no Programa de Aids: questões para a investigação em serviços de saúde no Brasil. Cad Saúde Pública. 2004;20(Supl 2):S310$\mathrm{S} 21$.

7. Melchior R, Nemes MIB, Basso CR, Castanheira ERL, Alves MTSSB, Buchalla, CM , Donini AA. Avaliação da estrutura organizacional da assistência ambulatorial em HIV/AIDS no Brasil. Rev Saúde Pública. 2006;40(1):143-51.

8. M elchior R. Avaliação da Organização da Assistência Ambulatorial A Pessoas Vivendo Com Hiv/Aids no Brasil. Análise de 322 serviços em 7 estados brasileiros 
(CE, MA, MS, PA, RJ , RS, SP. [tese]. São Paulo: Faculdade de Saúde Pública, Universidade de São Paulo; 2003

9. Nemes MIB, Carvalho HB, Souza MFMS. Antiretroviral therapy adherence in Brazil. AIDS. 2004;18(Suppl 3):5-20.

10. Mehta S, Moore RD, Graham, NMH. Potencial factors affecting adherence with HIV therapy. AIDS. 1997:11(14):1665-70,

11. Singh N, Squier C, Sivek C, Wagener M, Nguyen MH, Yu VL. Determinants of compliance with antiretroviral therapy in patients with human immunodeficiency virus: prospective assessment with implications for enhancing compliance. AIDS Care. 1996:8(3): 261-269.

12. Pratt J H, J ones JJ. Noncompliance with therapy: an ongoing problem in treating hypertension. Prim Cardiol. 1995;21(1):34-8.

13. Becker $\mathrm{MH}$. Patient adherence to prescribed therapies. Med Care. 1985:23(4):539-55

14. Castanheira ERL. Avaliação da assistência ambulatorial a pessoas vivendo com hiv/Aids em serviço públicos no estado de São Paulo: relações entre qualidade processo do trabalho. [tese] São Paulo: Faculdade de Medicina, Universidade de São Paulo; 2002.

15. Alves MTSSB. Avaliação da assistência ambulatorial à pessoas vivendo com HIV e Aids no Sistema Ú nico de Saúde: a situação do Maranhão. [tese]. São Paulo: Faculdade de Medicina, Universidade de São Paulo; 2003

16. Alencar TMD, Nemes MIB, Velloso MA. Transformações da 'Aids aguda' para a 'Aids crônica': percepção corporal e intervenções cirúrgicas entre pessoas vivendo com HIV e Aids . Ciênc Saúde Coletiva. 2008;13(6):1841-9. Disponível em: http://www.abrasco.org.br/cienciaesaudecoletiva/artigos/ artigo int.php?id artigo $=380$.

17. Santos C, Felipe $Y$, Braga P, Ramos D, Lima R, Segurado A. Self-perception of body changes in persons living with HIV/AIDS: prevalence and associate factors. AIDS. 2005;19(Suppl 4):S14-21.

18. Lignani J unior L, Greco DB, Carneiro M. Avaliação da aderência aos antiretrovirais em pacientes com infecção pelo HIV/AIDS. Rev Saúde Pública $2001 ; 35(4): 495-501$

19. Pinheiro CA, Carvalho-Leite J C, Drachler ML, Silveira VL. Factors associated with adherence to antiretroviral therapy in HIV/AIDS patients: a crosssectional study in Southern Brazil. Braz। Med Biol Res 2002; 35(10):1173-81.

20. Brigido LF, Rodrigues R, Casseb J, Oliveira D, Rossetti M, Menezes $P$, et al. Impact of adherence to antiretroviral therapy in HIV-1-infected patients at a university public service in Brazil. AIDS Patient Care STDS. 2001;15(11):587-93

21. Carvalho CV, Duarte DB, Merchán-Hamann E, Bicudo E, Laguardia J. Determinantes da aderência à terapia anti-retroviral combinada em Brasília, Distrito Federal, Brasil, 1999-2000. Cad Saúde Pública. 2003;19(5):593-604.

22. Hofer $C B$, Schechter $M$, Harrison LH. Effectiveness of antiretroviral therapy among patients who attend public HIV clinics in Rio de J aneiro, Brazil. J Acquir Immune Defic Syndr. 2004;36(4):967-71.

23. Bonolo PF, Cesar CC, Acurcio FA, Ceccato MG, Pádua CA, Álvares J, et al. Non-adherence among patients initiating antiretroviral therapy: a challenge for health professionals in Brazil. AIDS. 2005;19(Suppl 4):S5-13

24. Barroso PF, Schechter M, Gupta P, Bressan C, Bomfim A, Harrison LH. Adherence to antiretroviral therapy and persistence of HIV RNA in semen. J Acquir Immune Defic Syndr. 2003:32(4):435-40.

25. Moatti J P, Spire B, Kazatchkine M. Drug resistance and adherence to HIV/ AIDS antiretroviral treatment: against a double standard between the north and the south. AIDS. 2004; 18(Suppl 3):S55-61.

26. Programa Nacional de DST e Aids. Conceitos e recomendações básicas para melhorar a $80 \%$ adesão ao tratamento anti-retroviral. Boletim Epidemiológico. 1998;11(03). Semana Epidemiológica 22 a 34/98. Disponível em: http://www.Aids.gov.br.

27. Garcia R, Ponde M, Lima M, Souza AR, Stolze SM, Badaro R. Lack of effect of motivation on the adherence of HIV-positive/AIDS patients to antiretroviral treatment. BrazJ InfectDis. 2005; 9(6):494-9.
28. Gupta N, Silva AC, Passos LN. The role of integrated home-based care in patient adherence to antiretroviral therapy. Rev Soc Bras Med Trop. 2005;38(3):241-5

29. Oliveira LA, Landroni MAS, Silva NEK, Ayres J RCM. Humanização e cuidado: a experiência da equipe de um serviço de DST/Aids no município de São Paulo. Ciênc. Saúde Coletiva. 2005;10(3):689-98.

30. Nemes MIB, Basso CR, Castanheira ERL, Melchior R, Alencar TMD, Caraciolo J MM , Alves MTSSB. QU ALIAIDS - Avaliação e monitoramento da qualidade da assistência ambulatorial em Aids no SUS. 1. ed. Brasília: Ministério da Saúde, 2008. v.1. 100 p.

31. Nemes, MIB; Basso, CR; Castanheira, ERL; Melchior, R ; Alencar, TMD; Caraciolo, J M M ; Alves, MTSSB. QUALIAIDS - Avaliação e monitoramento da qualidade da assistência ambulatorial em Aids no SUS. 1. ed. Brasília: Ministério da Saúde, 2008. v.1.100 p.

32. Nemes, MIB; Alencar, TMD. Avaliação da assistência ambulatorial aos adultos vivendo com HIV/Aids QUALIAIDS, Relatório 2007/2008. Brasília: Ministério da Saúde, 27 p. 2008. Disponível em: http://sistemas.aids.gov.br/qualiaids/ Relat\%F3rio_Qualiaids_2008_PDF.pdf.

33. Brito AM, Szwarcwald CL; Castilho EA. Fatores associados à interrupção de tratamento anti-retroviral em adultos com AIDS. Rio Grande do Norte, B rasil, 1999-2002. Rev Assoc Med Bras. 2006;52(2):86-92.

34. J ordan M, Lopes J F, Okazaki E, Komatsu CL, Nemes MIB. Aderência ao tratamento anti-retroviral em Aids: revisão da literatura médica. In: Teixeira P R, Paiva V, Shimma $E$, organizadores. Tá difícil de engolir? Experiências de adesão ao tratamento anti-retroviral em São Paulo. São Paulo: NEPAIDS-SP, CRT-SP; 2000.

35. Bartlett, | A. Addressing the challenges of adherence. I Acquir Immune Defic Syndr. 2002; 29(Suppl 1): S2-S10.

36. Golin, CE, Liu, HH, Hays, RD, Miller, LG, Beck, CK, Ickovics, J, etal. A prospective study of predictors of adherence to combination antiretroviral medication.J Gen Intern Med. 2002;17(10):756-65.

37. Nemes MIB, Beaudoin J, ConwayS, Kivumbi G, Skjelmerud A, Vogel U. Evaluation of WHO's contribution to " 3 by 5". Genene: World Health Organization; 2006 Available from: www.who.int/hiv/topics/me/3 by5 evaluationreport. pdf.

38. The World Bank. Confronting Aids: public priorities In: A Global Epidemic. Oxford: University Press; 1997

39. Popp D, Fisher J D. First, do no harm: a call for emphasizing adherence and HIV prevention interventions in active antiretroviral therapy programs in the developing world. AIDS. 2002;16(4):676-8.

40. Miles K, Clutterbuck DJ , Seitio O, Sebego M A Riley A Antiretroviral treatmentrollout in a resource-constrained setting: capitalizing on nursing resources in Botswana. Bull World Health Organ, 2007;85(7):555-60.

41. Petersen ML, Boily M, Bastos FI. Assessing HIV resistance in developing countries: Brazil as a case study. Rev Panam Salud Publica. 2006;19(3):146-56.

42. Hacker MA, Kaida A, Hogg RS, Bastos FI. Os primeiros dez anos: conquistas e desafios do programa brasileiro de acesso ao manejo e cuidado integral do HIVI AIDS no Brasil, 1996-2006. Cad. Saúde Pública. 2007;.23(Supl 3):S345-S59.

43. Castilho EA, Scwarcwald CL, Bastos FI, Fonseca M GM. A Aids no Brasil: uma epidemia em mutação. Cad Saúde Publica. 2000;16(Supl 1):4-5. 